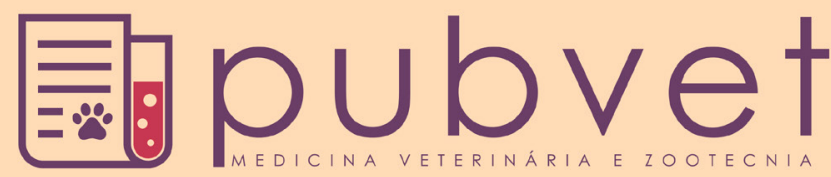

\title{
Estudo da variabilidade espacial da concentração de cobre e cádmio ao longo da margem do rio Meuse
}

\author{
Lourenço Manuel', João Domingos Scalon², Guido Gustavo Humada Gonzalez ${ }^{3}$, Juracy \\ Mendes Moreira ${ }^{4}$, Marcelo Carlos Ribeiro ${ }^{5}$, Aurélio Ferreira Melo ${ }^{6 *}$
}

${ }^{1}$ Doutorando em Estatística e Experimentação Agropecuária DES/UFLA. Lavras - MG: E-mail: zloremanuel@gmail.com

${ }^{2}$ Prof. Pos-Doutor. Prof. Departamento de Estatística DES/UFLA.E-mail: scalon@des.ufla.br

${ }^{3}$ Prof. Doutor. Universidad San Carlos - Asunción-Paraguay E-mail: gustavohumad@hotmail.com

${ }^{4}$ Prof mestre. Instituto Federal Goiano. Campus Rio Verde - GO E-mail: juracimendesmoreira@yahoo.com.br

${ }^{5}$ Doutorando em Estatística Aplicada e Biometria. Universidade Federal de Viçosa. MG. E-mail: marcelocarlosribeiro@hotmail.com

${ }^{6}$ Doutorando Instituto Federal Goiano. Campus Rio Verde - GO. E-mail: aurelioferreiramelo@hotmail.com

* Autor para correspondência

RESUMO. O conhecimento dos níveis de concentração de metais pesados no ambiente e sua disseminação no solo e nas águas são de primordial importância em estudos ambientais, por constituir um dos indicadores de "medição" do equilíbrio da biodiversidade e da estabilidade dos ecossistemas. O presente trabalho teve como objetivo, estudar a distribuição espacial de dois metais pesados (cobre e cádmio) ao longo das margens do rio Meuse, por forma a medir os níveis de contaminação por esses metais. Foram usados dados da concentração de $\mathrm{Cd}$ e $\mathrm{Cu}$ amostrados em 155 pontos georeferenciados. Estes dados foram obtidos no programa R no pacote "gstat" cuja base de dados denomina-se "Meuse". A metodologia de análise dos dados consistiu em ajustar semivariogramas para análise da dependência espacial, e foram ajustados os modelos esféricos e gaussiano para a concentração de Cobre e cádmio, respectivamente. O grau de dependência espacial foi classificado como forte para a concentração de Cobre e moderada para a concentração de cádmio. A estimação da concentração destes metais pesados em pontos não amostrados foi feita usando o método de krigagem produzindo mapas de distribuição espacial da concentração de cobre e cádmio que apresentaram um padrão similar entre si. Verificou-se que dentre os dois metais pesados, o Cobre apresenta concentrações muito altas que chegam a atingir os $130 \mathrm{mg} / \mathrm{Kg}$ de solo.

Palavras Chave: Metais pesados, geoestatística, semivariograma, krigagem

\section{Study of the spatial variability of copper and cadmium concentration along the Meuse River margin}

\begin{abstract}
The knowledge of the concentration levels of heavy metals in the environment and their dissemination in soil and water are of paramount importance in environmental studies, as one of the indicators of "measuring" the balance of biodiversity and the stability of ecosystems. The objective of this study was to study the spatial distribution of two heavy metals (Copper and Cadmium) along the banks of the Meuse River, in order to measure the levels of contamination by these metals. $\mathrm{Cd}$ and $\mathrm{Cu}$ concentration data were sampled at 155 georeferenced points. These data were obtained in the program $\mathrm{R}$ in the package "gstat" whose database is called "Meuse". The data analysis methodology consisted in adjusting semivariograms for spatial dependence analysis, and the spherical and Gaussian models were adjusted for the copper and cadmium concentration, respectively. The degree of spatial dependence was classified as strong for the copper concentration and moderate for the cadmium concentration. The estimation of the concentration of these heavy metals in non-sampled points was done using the kriging
\end{abstract}


method, producing spatial distribution maps of the copper and cadmium concentrations that presented a similar pattern. It was verified that of the two heavy metals, Copper has very high concentrations that reach $130 \mathrm{mg} / \mathrm{kg}$ of soil.

Keywords: Heavy metals, geostatistics, semivariogram, kriging

\title{
Estudio de la variabilidad espacial de la concentración de cobre y el cadmio a lo largo del mar del río Meuse
}

\begin{abstract}
RESUMEN. El conocimiento de los niveles de concentración de metales pesados en el medio ambiente y su diseminación en el suelo y en las aguas son de primordial importancia en estudios ambientales, por constituir uno de los indicadores de medición del equilibrio de la biodiversidad y de la estabilidad de los ecosistemas. El presente trabajo tuvo como objetivo, estudiar la distribución espacial de dos metales pesados (Cobre y Cadmio) a lo largo de las márgenes del río Meuse, para medir los niveles de contaminación por estos metales. Se utilizaron datos de la concentración de $\mathrm{Cd}$ y $\mathrm{Cu}$ muestreados en 155 puntos georeferenciados. Estos datos se obtuvieron en el programa $\mathrm{R}$ en el paquete "gstat" cuya base de datos se denomina "Meuse". La metodología de análisis de los datos consistió en ajustar semivariogramas para análisis de la dependencia espacial, y se ajustaron los modelos esféricos y gaussiano para la concentración de Cobre y Cadmio, respectivamente. El grado de dependencia espacial fue clasificado como fuerte para la concentración de Cobre y moderado para la concentración de Cadmio. La estimación de la concentración de estos metales pesados en puntos no muestreados, fue hecha usando el método de kriging produciendo mapas de distribución espacial de la concentración de Cobre y Cadmio que presentaron un patrón similar entre sí. Se verificó que entre los dos metales pesados, el Cobre presenta concentraciones muy altas que llegan a alcanzar los $130 \mathrm{mg} / \mathrm{Kg}$ de suelo.
\end{abstract}

Palabras clave: Metales pesados, geoestadísticos, semivariograma, krigado

\section{Introdução}

O rio Meuse com $950 \mathrm{~km}$ de extensão nasce na França, no planalto de Langres, Bassigny, atravessa a Bélgica, Países Baixos e segue em direção ao Mar do Norte. Possui uma vazão média de $400 \mathrm{~m}^{3} / \mathrm{s}$ e a área da bacia hidrográfica do Meuse é de $36.000 \mathrm{~km}^{2}$, sendo a maior área da bacia localizada na Valônia $\left(12.000 \mathrm{~km}^{2}\right)$, seguido pela França $\left(9.000 \mathrm{~km}^{2}\right)$, Holanda $\left(8.000 \mathrm{~km}^{2}\right)$, Alemanha $\left(2.000 \mathrm{~km}^{2}\right)$, além de outros países europeus. Classificado como um rio meandrante, é caracterizado por intensa deposição de sedimentos finos como silte e argila.

A qualidade do solo é fundamental ao desenvolvimento sustentável e à preservação dos ecossistemas e da biodiversidade. Nesse contexto, a contaminação do solo por metais pesados, põe em risco sua capacidade produtiva e o equilíbrio dos ecossistemas. Assim, o conhecimento dos níveis de concentração dos metais pesados no solo de uma determinada região ou em ambiente aquífero é bastante importante, pois permite medir os níveis de contaminação por este ou aquele elemento químico (Valladares et al., 2009).

A estatística clássica pressupõe que as observações feitas numa determinada variável são independentes. Em caso de estudos envolvendo ciências da terra, essa pressuposição não é verdadeira, devido ao fato de se assumir que a distribuição espacial de pontos de observação tenha correlação, isto é, existe uma dependência espacial, portanto, em estudos de ciências de solos, as técnicas da estatística clássica tornam-se inadequadas, dando necessidade ao uso de outras técnicas que incorporem as relações espaciais entre as observações. Tais técnicas são preconizadas pela geoestatística, que para além de fazer a análise da distribuição estatística dos dados coletados, incorpora também as relações espaciais entre estes na forma de correlação entre pontos amostrados. Trangmar et al. (1986) afirmam que amostras mais próxima dentro de uma mesma mancha de solo são mais parecidas do que as mais distantes. Por estas razões (Goovaerts, 1997) afirma que os problemas da ciência da terra são efetivamente analisados atualmente por técnicas da geoestatística, quando a interpretação da distribuição espacial dos dados tem forte impacto sobre os resultados e a tomada de decisão (Fadigas, 2002, Camargo et al., 2003).

O presente trabalho teve como objetivo estudar a distribuição espacial da concentração de dois 
metais pesados (cádmio e cobre) ao longo das margens do rio Meuse para avaliar os níveis de contaminação por estes metais que causam grande risco na alteração do ecossistema e na biodiversidade.

\section{Material e Métodos}

O conjunto de dados usados no presente trabalho foi extraído de uma base de dados do programa $\mathrm{R}$ no pacote gstat. $\mathrm{O}$ banco de dados denomina-se Meuse e contém informação de 155 pontos georeferenciados onde se mediu a concentração de 4 metais pesados (cádmio, cobre, níquel e chumbo). A amostragem dos pontos foi feita num plano bidimensional na parte superficial do solo usando um intervalo de amostragem de 15 $\mathrm{m} \times 15 \mathrm{~m}$. Estes metais são transportados pelo rio Meuse e sedimentados ao longo de suas margens. No presente estudo só foram usados dados da concentração de dois metais pesados, o cádmio e cobre. A figura 1 mostra a região de estudo e os pontos amostrados.

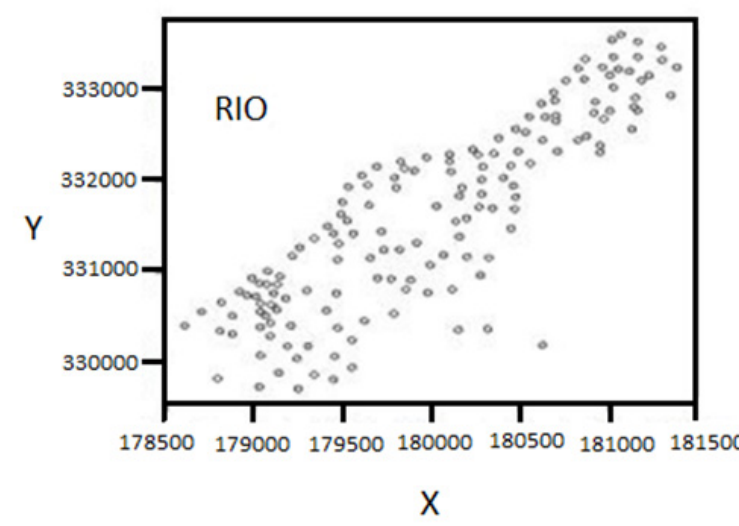

Figura 1. Região estudada e os pontos amostrados

Em problemas de ciências da terra como em ciência de solos, as observações de uma determinada variável apresentam uma correlação espacial. Considere uma característica do solo $Z$, tal como a concentração de cobre, que pode variar continuamente num espaço geográfico, em função das coordenadas geográficas. Cada valor observado $z(x i)$ nos locais $x i, i=1,2 \ldots, n$, em que $x i$ denota coordenada geográfica em duas dimensões, é considerado uma realização da variável aleatória $Z(x)$. O conjunto das variáveis aleatórias $Z(x 1) \ldots Z(x n)$ constitui um processo estocástico.

\section{Análise da dependência espacial}

A representação da estrutura da dependência espacial destas variáveis aleatórias no espaço, quando x varia, é dada pelo semivariograma
(Mello et al., 2005). Na figura 2 está apresentado um semivariograma típico com os seus parâmetros principais nomeadamente o efeito pepita $(\mathrm{Co})$, patamar $(C+C o)$ e alcance (a).

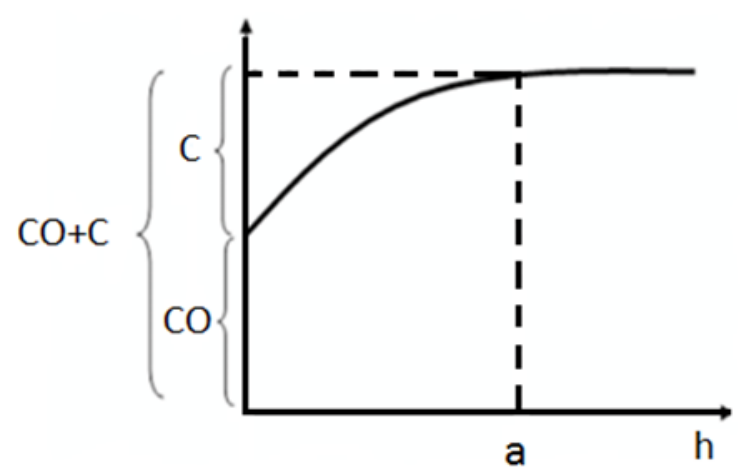

Figura 2. Semivariograma típico com os principais parâmetros

A semivariância é a "ferramenta" estatística que indica o valor da dependência espacial entre amostras de uma determinada variável em função da distância entre elas. Na ausência de tendência, a semivariância é estimada com base na equação 1 também conhecida como estimador de Matheron (Gotway and Waller, 2004).

$$
\hat{\gamma}(h)=\frac{\sum_{h=i}^{N(h)}[z(x)-z(x+h)]^{2}}{2 N(h)}
$$

Em situações em que a variável em estudo apresenta valores atípicos "outliers", o cálculo da semivariância é feito com base num estimador robusto proposto por Cressie e Hawikins (Cressie, 2015) dado pela equação 2 .

$$
=\frac{\left\{\frac{1}{N(h)} \sum_{N(h)}[z(x)-z(x+h)]^{1 / 2}\right\}^{4}}{2\left(0,457+\frac{0,494}{N(h)}\right)}
$$

Nas equações 1 e 2 , h é a distância entre dois pontos, $N(h)$ é o número de pares de pontos separados pela distância $h, z(x)$ é o valor da variável de interesse no ponto $x, z(x+h)$ é a realização de $Z$ no ponto $x+h$ e $\gamma(h)$ é a estimativa da semivariância entre pares de pontos separados pela distância $h$.

O grau de dependência espacial $(G D)$ foi determinado como a proporção do efeito pepita $(C o)$ no patamar $(C o+C)$ (equação 3$)$. Segundo Guimarães (2004) se o $G D<0.25$, a variável apresenta forte dependência espacial, se $0.25<$ $G D<0.75$, a dependência espacial é moderada, se $0.75<G D<1$ a dependência espacial é 
fraca e quando $G D=1$ a variável em estudo não apresenta dependência espacial.

$$
G D=\frac{C o}{C+C o}
$$

\section{Krigagem}

A estimação dos valores da variável de interesse em pontos ou locais não amostrados na região de estudo foi feita usando o método de krigagem (equação 4). Este método, comparado com outros métodos que se baseiam em estimadores lineares (IQD, ID etc) é o melhor, pois garante que em média, as estimativas serão não tendenciosas (Vieira et al., 2002).

$$
\begin{aligned}
\sum_{i=1}^{n} \gamma_{i} \gamma\left(x_{i}-x_{j}\right) & +\mu \\
= & \gamma\left(x_{i}, x_{0}\right),
\end{aligned}
$$

$\sum_{i=1}^{n} \gamma_{i}=1$

Em que $\gamma(x i, x j)$ é o valor da semivariância estimada, usando o modelo ajustado ao semivariograma, correspondente à distância entre os pontos localizados na posição $x_{i}$ e $x_{j}\left(x_{i}-x_{0}\right)$ é a semivariância correspondente à distância entre o ponto localizado na posição $x_{i}$ e o ponto a ser krigado $\left(x_{0}\right), \mu$ é o multiplicador de Lagrange associado com a minimização da variância e $\gamma_{i}$ são os pesos dos valores de $Z$ usados para obter a estimativa no ponto a ser krigado $\left(x_{0}\right)$. A estimativa $Z\left(x_{0}\right)$ do ponto a ser krigado foi obtida segundo a equação 5 .

$$
Z\left(x_{0}\right)=\sum_{i=1}^{n} \beta_{i} Z_{i}
$$

Onde $Z\left(x_{0}\right)$ é a estimativa do ponto a ser krigado, $\beta_{i}$ são as estimativas dos pesos obtidas no sistema de equações da equação 4 e $Z_{i}$ valores da variável nos pontos amostrados.

\section{Resultados e Discussão}

Com base na estatística descritiva (Tabela 1) verificou-se que a concentração mínima do cádmio foi de $0,2 \mathrm{mg} / \mathrm{kg}$ de solo e o máximo de $18 \mathrm{mg} / \mathrm{kg}$, com uma concentração média de $3,25 \mathrm{mg} / \mathrm{kg}$ e uma mediana de $2,1 \mathrm{mg} / \mathrm{kg}$. Para o cádmio a concentração mínima foi de $14 \mathrm{mg} /$ $\mathrm{kg}$ e a máxima de $128 \mathrm{mg} / \mathrm{kg}$ com uma concentração média de cerca de $40,31 \mathrm{mg} / \mathrm{kg}$ e uma mediana de $31 \mathrm{mg} / \mathrm{kg}$. Os dados da

concentração do cádmio apresentaram menor dispersão $(S=3,52)$ relativamente a concentração de cobre $(S=23,68)$. Em ambas variáveis, o valor da mediana é inferior a média o que mostra que há indícios de que os dados apresentam uma distribuição assimétrica à direita, com presença de alguns "outliers" como pode ser visualizado pelo gráfico de "box-plot" (igura 3).

Tabela 1. Análise descritiva da concentração de cádmio e Cobre ( $\mathrm{mg} / \mathrm{kg}$ do solo)

\begin{tabular}{ccc}
\hline & Cádmio & Cobre \\
\hline $\mathrm{N}$ & 1,55 & 155 \\
Mínimo & 0,2 & 14 \\
Máximo & 18,1 & 128 \\
Média & 3,25 & 40,31 \\
Mediana & 2,1 & 31 \\
Variância & 12,42 & 560,73 \\
Desvio padrão & 3,52 & 23,68 \\
Assimetria & 1,76 & 1,39 \\
Curtose & 3,05 & 1,22 \\
\hline
\end{tabular}

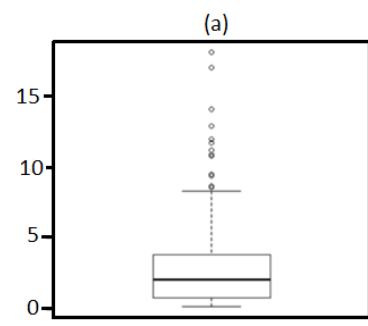

Dados

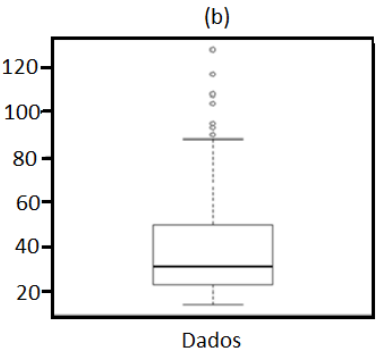

Figura 3. Gráfico box-plot da concentração de (a) cádmio (b) cobre

\section{Estudo da dependência espacial}

Os semivariogramas experimentais da concentração de cádmio e Cobre foram construídos usando o estimador robusto de Cressie e Hawikins (equação 2) devido a presença de valores atípicos "outliers" (Cressie, 2015) conforme ilustram os gráficos "box-plot" (Figura 3). Em ambos semivariogramas assumiu-se isotropia do fenômeno, e foram ajustados modelos esféricos e gaussiano para a concentração de cobre e cádmio, respectivamente (Figura 4). Quanto ao grau de dependência espacial, verificou-se que a concentração de Cobre apresentou uma forte dependência espacial, pois o efeito pepita representa cerca de $22 \%$ do patamar. Para a concentração do cádmio, o grau de dependência espacial foi considerado moderado, uma vez que o efeito pepita do modelo gaussiano representa cerca de $27 \%$ do patamar, ou seja, o grau de 
dependência espacial para a concentração de cádmio encontra-se no intervalo de 0.25 e 0.75 .

Pelo método de krigagem, foram estimados valores da concentração de cádmio e cobre nos pontos onde não foi feita a amostragem, gerando mapas da distribuição espacial da concentração de cádmio e cobre (Figura 4).
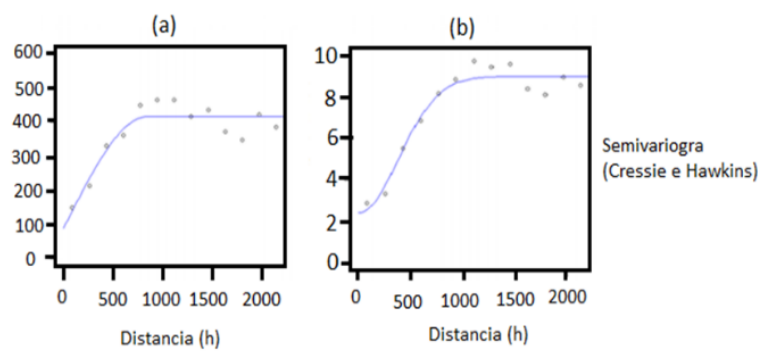

Figura 4. Semivariogramas ajustados (a) $\mathrm{Cu}$ modelo esférico (b) Cd modelo gaussiano.

Tabela 2. Estimativas dos parâmetros dos modelos ajustados (esférico para $\mathrm{Cu}$ e gaussiano para $\mathrm{Cd}$ )

\begin{tabular}{|c|c|c|c|c|}
\hline Variável & $\begin{array}{l}\text { Efeito } \\
\text { pepita } \\
\left(m g^{2}\right. \\
\left./ \mathrm{kg}^{2}\right) \\
\end{array}$ & $\begin{array}{l}\text { Contribuição } \\
\left(m g^{2} / k g^{2}\right)\end{array}$ & $\begin{array}{c}\text { Patamar } \\
\left(m g^{2}\right. \\
\left./ k g^{2}\right)\end{array}$ & $\begin{array}{c}\text { Alcance } \\
m\end{array}$ \\
\hline $\begin{array}{l}\text { Concentração } \\
\text { de cádmio }\end{array}$ & 2,39 & 6,66 & 9,05 & 939,46 \\
\hline $\begin{array}{l}\text { Concentração } \\
\text { de cádmio }\end{array}$ & 89,90 & 327,50 & 417,40 & 843,70 \\
\hline
\end{tabular}

Com base no modelo gaussiano, verificou-se que para a concentração do cádmio, o alcance obtido foi de $940 \mathrm{~m}$ enquanto que para o cobre, o modelo esférico indicou um alcance de aproximadamente $844 \mathrm{~m}$. Isto significa que amostras retiradas a uma distância inferior a 940 m e $844 \mathrm{~m}$ para o cádmio e cobre respectivamente, apresentam uma dependência espacial entre si. Este fato permite afirmar que os métodos de análise de dados baseados na estatística clássica, só serão válidos se a amostragem for feita a distâncias superiores a $940 \mathrm{~m} \mathrm{e} 844 \mathrm{~m}$, para o cádmio e cobre, respectivamente (Figura 2).

Com base na figura 5 pode-se notar que a distribuição espacial da concentração de cádmio e cobre, apresentam um padrão similar entre si, isto é, nos dois casos, maiores concentrações dos metais pesados são encontrados nas regiões norte e noroeste (dentro do rio - Figura 1), estando acima de $100 \mathrm{mg} / \mathrm{kg}$ de solo para o cobre e acima de $15 \mathrm{mg} / \mathrm{kg}$ de solo para o cádmio. Na zona central da região do estudo, a concentração dos metais pesados é ligeiramente baixa (cerca de 20 a $40 \mathrm{mg} / \mathrm{kg}$ de solo para o cobre e 0 a $5 \mathrm{mg} / \mathrm{kg}$ de solo para o cádmio). Na zona sul e leste, a concentração de cobre varia na faixa de 40 a 60 $\mathrm{mg} / \mathrm{Kg}$ de solo enquanto que a concentração de cádmio encontra-se em torno de $5 \mathrm{a} 10 \mathrm{mg} / \mathrm{kg}$ de solo, embora exista uma pequena área com concentração muito alta (acima de $15 \mathrm{mg} / \mathrm{kg}$ de solo). Cobre, e dependência moderada para a concentração do cádmio.
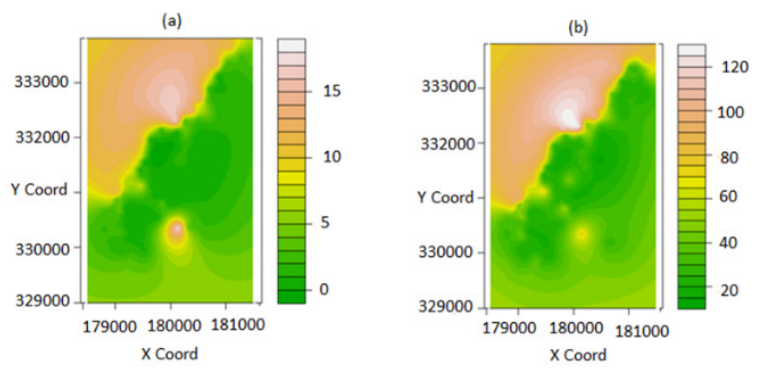

Figura 5. Distribuição espacial da concentração de (a) Cd (b) $\mathrm{Cu}$ na região de estudo.

\section{Conclusão}

As concentrações de cádmio e cobre ao longo das margens do rio Meuse apresentam-se espacialmente estruturadas, com forte grau de dependência espacial para a concentração do Cobre, e dependência moderada para a concentração do cádmio. O padrão de distribuição espacial de cádmio na região de estudo é idêntico ao padrão da concentração de $\mathrm{Cu}$. O cobre é o metal pesado que causa maior poluição na região de estudo. Assim, devem ser tomadas medidas de visem reduzirem os atuais níveis de contaminação das águas do rio.

\section{Agradecimentos}

Ao Conselho Nacional de Desenvolvimento Científico (CNPq) pela bolsa de estudos.

\section{Referências Bibliográficas}

Camargo, O. A., Borba, R. P. \& Alleoni, L. R. F. 2003. Metais pesados: da cosmogênese aos solos brasileiros. Congresso Brasileiro de Ciência do Solo. Universidade Federal de Viçosa, Viçosa.

Cressie, N. 2015. Statistics for spatial data. John Wiley \& Sons, New York.

Fadigas, F. S. 2002. Estimativas das concentrações naturais (pseudo-total) de $\mathrm{Cd}$, $\mathrm{Co}, \mathrm{Cr}, \mathrm{Cu}, \mathrm{Ni}, \mathrm{Pb}$ e $\mathrm{Zn}$ em solos brasileiros e proposição de valores de referência utilizando técnicas da estatística multivariada.

Goovaerts, P. 1997. Geostatistics for natural resources evaluation. Oxford University Press on Demand, New York. 
Gotway, C. A. \& Waller, L. A. 2004. Applied spatial statistics for public health data. John Wiley \& Sons, New York.

Guimarães, E.C. 2004. Geoestatística básica e aplicada. Apostila. Uberlândia.

Mello, J. M., Ferreira, B. J. L., Silva, O. M. \& Justiniano, R. P. 2005. Estudo da dependência espacial de características dendrométricas para Eucalyptus grandis. Cerne, 11, 113-126.

Trangmar, B. B., Yost, R. S. \& Uehara, G. 1986. Application of geostatistics to spatial studies of soil properties. Advances in Agronomy, 38, 4594.

Valladares, G. S., Azevedo, E. C., Camargo, O. A., Grego, C. R. \& Rastoldo, M. C. S. 2009.
Variabilidade espacial e disponibilidade de cobre e zinco em solos de vinhedos e adjacências. Bragantia, 68, 733-742.

Vieira, S. R., Millete, J., Topp, G. C. \& Reynolds, W. D. 2002. Handbook for geostatistical analysis of variability in soil and climate data. Tópicos em ciência do solo, 2, 1-45.

\section{Article History:}

Received 2 May 2017

Accepted 25 May 2017

Available on line 30 June 2017

License information: This is an open-access article distributed under the terms of the Creative Commons Attribution License 4.0, which permits unrestricted use, distribution, and reproduction in any medium, provided the original work is properly cited 